\title{
Comparison of maternal and neonatal outcome in elective lower segment cesarean section done at 38 and 39 weeks
}

\author{
Gisi Sebastian $^{1}$, Seetesh Ghose ${ }^{1 *}$, P. Soundararajan ${ }^{2}$
}

\author{
${ }^{1}$ Department of Obstetrics and Gynecology, ${ }^{2}$ Department of Pediatrics, Mahathma Gandhi Medical College and \\ Research Instituite, Puducherry, Tamil Nadu, India
}

Received: 19 January 2017

Accepted: 28 February 2017

\author{
*Correspondence: \\ Dr. Seetesh Ghose, \\ E-mail: seetesh@mgmcri.ac.in
}

Copyright: (c) the author(s), publisher and licensee Medip Academy. This is an open-access article distributed under the terms of the Creative Commons Attribution Non-Commercial License, which permits unrestricted non-commercial use, distribution, and reproduction in any medium, provided the original work is properly cited.

\section{ABSTRACT}

Background: Time to perform elective LSCS is a relevant issue related to mother and foetus. LSCS can be done from 37 weeks onwards because foetal lungs mature by then. But foetuses born at 37 weeks of gestation have more risk of developing respiratory problems, transient tachypnoea of new born, hypothermia, hypoglycaemia and NICU admission.The purpose of this study is to find out appropriate gestational age at which elective LSCS can be performed without adverse maternal and neonatal outcome.

Methods: This is an observational comparative study done on 209 antenatal women who underwent elective caesarean section from December 2014 to January 2016.Patients were divided into two groups after taking consent. Maternal parameters like formation of lower uterine segment need for blood transfusion. Neonatal parameters like Apgar score, respiratory distress syndrome and NICU admission with indication and duration were analyzed by chisquare test. Gestational Age and Pre-Op Hb and Post-Op Hb were analyzed by independent 't'test.

Results: In this study $55 \%$ of the pregnant women belong to 38 weeks, $45 \%$ belongs to 39 weeks. The formation of lower uterine segment was statistically significant and the need for blood transfusion was slightly increased at 39 weeks. But there was no significant difference in Apgar score, Respiratory distress, NICU admission comparing both study groups.

Conclusions: Elective caesarean section can be done safely at 38 weeks without affecting maternal and perinatal outcome.

Keywords: Elective caesarean section, Maternal outcome, Neonatal outcome

\section{INTRODUCTION}

Caesarean delivery is defined as an operative procedure in which fetus delivered through an incision in the abdominal wall (laparotomy) and the uterine wall (hysterotomy). This excludes delivery through an abdominal incision of a fetus, lying free in the abdominal cavity following the uterine rupture or in secondary abdominal pregnancy. ${ }^{1}$ Elective caesarean section means when the operation is done at a prearranged time during pregnancy to ensure the best quality of obstetrics, anesthesia, neonatal resuscitation and nursing services prior to the onset of labor. This operation is done about 1 week prior to the expected date of confinement. The common indications of caesarean section are, where vaginal delivery is not possible and foetal distress (non reassuring foetal heart rate). ${ }^{2}$ Respiratory distress syndrome is a common problem in premature babies. Respiratory distress syndrome occurring after elective LSCS is termed as iatrogenic RDS. ${ }^{3}$

Gestational age at the time of elective caesarean section also plays an important role in neonatal respiratory morbidity. Tita et al reported that neonatal morbidity is high in those babies born by elective LSCS done before 39 weeks. $^{4}$ Parilla et al observed that elective LSCS 
before 37 weeks has complications like pneumothorax and pulmonary hemorrhage. ${ }^{5}$ According to ACOG , it is estimated that $2.5 \%$ of all births in the United States are caesarean delivery at maternal request without any valid reason and the risk of respiratory distress syndrome, transient tachypnoea of new born, persistent pulmonary hypertension is more in elective LSCS done prior to 39 weeks. ${ }^{6}$ That's why elective LSCS is not performed before 39 weeks unless it is warranted either for maternal or foetal cause like severe PIH, severe IUGR etc.

According to Christie L Roberts et al and Cohen et al, there is $12 \%$ chance of spontaneous labour at 37-38 weeks for a women before planned non emergent elective caesarean section at $39-41$ weeks. $^{7,8}$ Because the incidence of maternal morbidity is high in emergency caesarean deliveries, by doing caesarean delivery at 38 completed weeks we can avoid the planned caesarean deliveries becoming an emergency event,(if enters into labour).

Thus, this study was conducted to find out appropriate gestational age at which elective LSCS can be performed without adverse maternal and neonatal outcome.

\section{METHODS}

It is an observational comparative study carried out from December, 2014 to January, 2016. Study population comprised all antenatal women admitted and undergoing planned caesarean section at 38 and 39 weeks of gestation.

\section{Inclusion criteria}

- Patients undergoing elective LSCS with

- Malpresentation

- Conceived after ART

- CPD

- Gravid women with previous caesarean section not willing to undergo VBAC or requesting for repeat caesarean section

- Bad obstetric history

\section{Exclusion criteria}

- Pregnancy with medical disorders like - gestational hypertension, preeclampsia, chronic hypertension, gestational or pre gestational diabetes, other medical conditions like cardiac, renal, thyroid, autoimmune disease or a previous still birth

- High risk pregnancy like IUGR, multiple gestation, placenta previa, abruption placentae

- Non reassuring FHR

Sampling procedure adopted was universal sampling on two groups.

- $\quad$ Elective LSCS at $38-38+6$ weeks.

- Elective LSCS at 39-39+6 weeks.
Sample size was; all the antenatal women undergoing elective LSCS and fulfilling the inclusion criteria during study period.

This study was started with human ethical committee approval. During the study period consent was obtained from all the antenatal women who fulfilled the inclusion criteria and underwent elective caesarean section . They were divided into two group based on gestational age. One group had antenatal women of gestational age 38 $38+6$ and second group had antenatal women of gestational age of 39-39+6. Group one had 115 patients and group two had 94 patients. Following parameters were assessed.

\section{Parameters to assess maternal outcome}

- Gestational age

- Pre op haemoglobin

- Post op haemoglobin

- Formation of lower uterine segment

- Post-partum haemorrhage

- Need for blood transfusion

\section{Parameters to assess neonatal outcome}

- $\quad$ APGAR score at 1 and 5 minutes

- Transient tachypnoea of new born

- Respiratory distress syndrome

- NICU admissions with indication.

\section{Data collection}

All data were entered into excel (MS excel 2011) Privacy and confidentiality was maintained. All patient identifiable numbers and information was stripped and replaced by anonymous numbers.

\section{Statistical analysis}

The quantitative variables like gestational age, pre op $\mathrm{Hb}$ and post op $\mathrm{Hb}$ were computed in mean and standard deviation and analyzed by independent ' $t$ 'test. The maternal and neonatal outcome variables like formation of lower uterine segment, need for blood transfusion, Apgar score, NICU admission with indication were computed for frequency and percentage and analyzed by chi-square test or fisher exact test.

\section{RESULTS}

During the study period total number of deliveries were 2313 in that $975(42.2 \%)$ were caesarean section, of that $209(30.6 \%)$ were elective LSCS and $676(69.4 \%)$ were emergency LSCS. In that 209 who underwent elective LSCS, 115 patients were at 38 weeks (55\%), and 94 patients were at 39 weeks (45\%). In our observation primigravida were $44.4 \%$ in 38 weeks and $56.4 \%$ in 39 weeks respectively. Multigravida was $53 \%$ in 38 weeks 
and $42.5 \%$ in 39 weeks respectively. Grand multipara was $2.6 \%$ at 38 weeks and $1.1 \%$ at 39 weeks respectively. The difference between primigravida and grand multi was comparable and whereas in multigravida it was statistically significant ( $\mathrm{p}$ value 0.0367 ). The common indication for which primigravida underwent elective
LSCS was Malpresentation. In which 70 patients had breech presentation and 34 had transverse lie, whereas in multigravida and grand multigravida the common indication is previous LSCS at maternal request (Table $1)$.

Table 1: Patient Profile

\begin{tabular}{|llll|}
\hline Period of gestation & 38 weeks & 39 weeks & P Value \\
\hline Parity & & & \\
\hline Primi & $51(44.4 \%)$ & $53(56.4 \%)$ & 0.8445 \\
\hline Multi (2-3) & $61(53 \%)$ & $40(42.5 \%)$ & $0.0367 *$ \\
\hline Grand multi (>4) & $3(2.6 \%)$ & $1(1.1 \%)$ & 0.3173 \\
\hline Indications of LSCS & & & Grand multigravida \\
\hline & Primigravida & Multigravida & 0 \\
\hline Malpresentation & 104 & 0 & 4 \\
\hline Previous LSCS at maternal request & 0 & 101 & \\
\hline
\end{tabular}

$P$ value $<0.05$ is significant.

In this study $14(12.1 \%)$ at 38 weeks had thinned out lower uterine segment whereas $16(17 \%)$ at 39 weeks which was not statistically significant. But 77 patients at 38 weeks and 47 patients at 39 weeks, lower uterine segment was not formed which was statistically significant ( $p$ value 0.0071 ). In this study, $16(13.9 \%)$ patients received blood transfusion in 38 weeks and 14 $(14.9 \%)$ patients in 39 weeks. Although it seems requirement for blood transfusion at 38 weeks was more in comparison to 39 weeks, it was not statistically significant. The mean preoperative $\mathrm{Hb} \%$ were $10.3 \mathrm{~g} \%$ and $10.1 \mathrm{~g} \%$ in women who underwent elective LSCS $(\mathrm{p}=0.283)$ at 38 and 39 weeks respectively. Similarly, the mean postoperative $\mathrm{Hb} \%$ were $9.3 \mathrm{~g} \%$ and $8.95 \mathrm{~g} \%$ for 38 and 39 weeks respectively $(\mathrm{p}=0.066)$. There was no significant difference in preoperative and post-operative mean haemoglobin (Table 2).

Table 2: Maternal Outcome.

\begin{tabular}{|c|c|c|c|}
\hline & \multicolumn{2}{|c|}{ Period of gestation } & \multirow{2}{*}{ P value } \\
\hline & 38 weeks & 39 weeks & \\
\hline \multicolumn{4}{|c|}{ Formation of lower uterine segment } \\
\hline Not well formed & $77(67 \%)$ & $47(50 \%)$ & $0.0071 *$ \\
\hline Well formed & $24(20.9 \%)$ & $31(33 \%)$ & 0.3452 \\
\hline Thinned out & $14(12.1 \%)$ & $16(17 \%)$ & 0.7153 \\
\hline Total & $115(100 \%)$ & $94(100 \%)$ & \\
\hline \multicolumn{4}{|c|}{ Need for blood transfusion } \\
\hline No & $99(86.1 \%)$ & $80(85.1 \%)$ & 0.1556 \\
\hline Yes & $16(13.9 \%)$ & $14(14.9 \%)$ & 0.7153 \\
\hline Total & $115(100 \%)$ & $94(100 \%)$ & \\
\hline \multicolumn{4}{|c|}{ Comparison of preoperative and post operative HB \% } \\
\hline Pre OP HB & 10.3 & 10.1 & 0.283 \\
\hline Post OP HB & 9.31 & 8.95 & 0.066 \\
\hline
\end{tabular}

$P$ value $<0.05$ is significant

In our observation $1(0.9 \%)$ at 38 weeks and $4(4.3 \%)$ at 39 weeks babies were having Apgar score <7. But it was not statistically significant. This table shows that totally 3 babies had respiratory distress syndrome of which 2 were in 39 weeks and 1 was in 38 weeks. But the difference was not significant. The need for NICU admission was 
compared in both groups. 2 babies from 38 weeks needed NICU admission (1.7\%). One due to meconium aspiration and another one due to congenital heart disease
(Tricuspid Artesia) detected after delivery. At 39 weeks, also 2 babies needed NICU admission (2.1\%) due to meconium aspiration syndrome. The difference was also not significant (Table 3).

Table 3: Neonatal outcome.

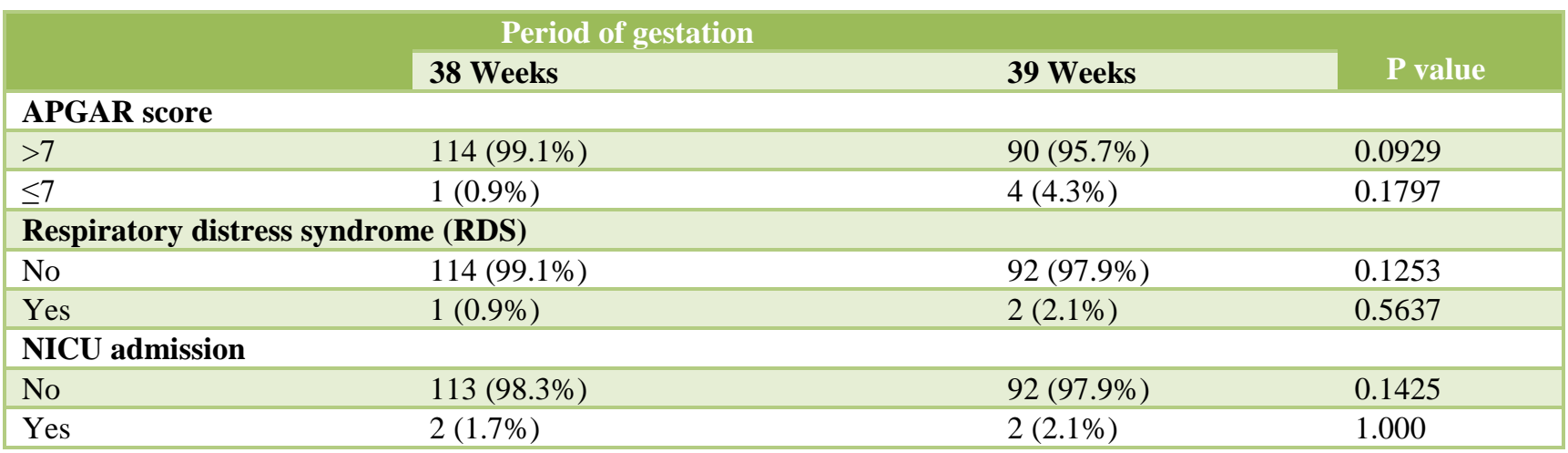

$P$ value $<0.05$ is significant

\section{DISCUSSION}

The rate of caesarean section over a period of time has increased globally in developed and developing countries. ${ }^{4}$ In comparison with elective LSCS done at 39 weeks, those underwent elective LSCS at 38 weeks had increased risk of poor maternal and neonatal outcome. The problems associated with neonates who born $<39$ weeks were, respiratory complications and admission in NICU. American College of Obstetrician and Gynaecologists states that for performing elective LSCS at 39 weeks or more, the date has to be confirmed with last menstrual period and 1 st trimester ultrasound. ${ }^{6}$ There are some studies which shows that even if we wait till 39 weeks for performing elective LSCS, it won't decrease the chance of adverse maternal and neonatal outcome. The main maternal complications of elective LSCS are uterine atonicity, post-partum hemorrhage, need for hysterectomy due to $\mathrm{PPH}$, increased hospital stay for $>5$ days, surgical site complications (endometriosis, wound complications) etc. It is more common with elective LSCS $<38$ weeks. ${ }^{4,9,10}$ But elective LSCS at 39-40 weeks are also having adverse effects like sudden unexplained fetal death, which increases after 37 weeks. ${ }^{10-12}$

In the present study among total elective LSCS, $115(55 \%)$ patients underwent elective LSCS at 38 weeks and $94(45 \%)$ patients at 39 weeks. Similarly TITA et al in their study $29.5 \%$ underwent elective LSCS at 38 weeks and $49.1 \%$ at 39 weeks. ${ }^{4}$ Glavind et al, showed that 635 patients at 38 weeks and 637 patients at 39 weeks underwent elective LSCS during the study period. ${ }^{13}$ According to Ghartey out of 2273 deliveries $51 \%$ underwent elective LSCS at 38-39 weeks. ${ }^{14}$ Similarly in a study by Emily Doan out of fourteen thousand four hundred and forty seven elective LSCS, $59.9 \%$ underwent elective LSCS before 39 weeks. ${ }^{15}$

In the present study $49.8 \%$ were primigravida, $48.3 \%$ were multigravida, and $1.9 \%$ were grand multigravida who underwent elective LSCS during the study period. But according to Glavind et al only $20 \%$ were primigravida and rest were multigravida.13But study done by Emily Doan and Wilmink et al observed that in their study there were $30 \%$ primigravida and $70 \%$ multigravida. It shows the increasing trend of elective LSCS in primigravida. ${ }^{15,16}$

In our present study out of 104 primigravida, 70 patients had breech presentation and 34 were having transverse lie. In multigravida and grand multigravida the indication for Elective LSCS was Previous LSCS at Maternal request. This was supported by the studies done by Sperling et al, Dahlgren et al, Mazzoni et al and Hourani et al. All these studies showed that the newer indication which has come in the developed world is maternal request, and the rate of primigravida undergoing caesarean section because of Malpresentation also has increased. ${ }^{17-20}$ Mylonas et al states that the anxiety of vaginal delivery which is called as Tocophobia also plays a major role in increasing number of elective LSCS. ${ }^{21}$

In present study $14(12.2 \%)$ at 38 weeks and $16(17.0 \%)$ patients had thinned out lower uterine segment. This was not statistically significant. This shows the increased risk of patient go into spontaneous labour in primigravida and increased chance of scar dehiscence in case of previous LSCS which increases the rate maternal morbidity. But $67 \%$ patients at 38 weeks and $50 \%$ patients at 39 weeks, lower uterine segment was not formed which was statistically significant. No other study is available for comparison. 
There was $1 \%$ increase in chance of need for blood transfusion found in present study at 39 weeks. 16 patients needed blood transfusion, which was $13.9 \%$ at 38 weeks and 14 patients in 39 weeks, which was $14.9 \%$ which was not statistically significant. In a study by Glavind et al, $1.4 \%$ patients needed blood transfusion who underwent elective LSCS at 38 weeks than $1.9 \%$ at 39 weeks.13Although Tita et al and Chiossi et al concluded that those patients underwent elective LSCS at 38 weeks needed more blood transfusion than 39 weeks, but it was not statistically significant. ${ }^{9,10}$ The increased rate of blood transfusion in our study was due to pre existing low haemoglobin.

In the present study $4.3 \%$ babies had low Apgar score at 39 weeks and it was due to increased incidence of meconium stained liquor. Whereas only $0.9 \%$ from 38 weeks had low Apgar score. Glavind et al, he observed that there is increased chance of less APGAR with increased gestational age. In this study he showed that $3.1 \%$ at 38 weeks had less Apgar score and $3.8 \%$ at 39 weeks.13Chiossi et al observed that the chance of low Apgar score was more with decreasing gestational age. That is $0.1 \%$ and $0.09 \%$ in 38 weeks and 39 weeks respectively.10Hourani et al, Bakr et al and Okeke et al in their study observed that the risk of low APGAR score decrease with increase in gestational age. ${ }^{20-23}$ Wilmink et al states that only after 40 weeks the APGAR score is $100 \%$ good < 40 weeks babies had minor risk of low APGAR score which was not statistically significant. ${ }^{16}$

In the present study $0.9 \%$ at 38 weeks and $2.1 \%$ at 39 weeks babies had respiratory distress syndrome out of 209 women who underwent elective LSCS. But Zonardo et al concluded that there is $12.9 \%$ chance of respiratory distress syndrome at $\leq 38$ weeks and at $\geq 39$ weeks it was $1.12 \%$ which was comparatively lesser with advanced gestational age.25Similar reports was showed by Tita et al, Emily Doan, Hourani et al, Wilmink et al, Chiossi et al. They all showed that the rate of respiratory distress decreases with increased gestational age. ${ }^{4,10,15,16,20}$ In present study the increased rate of respiratory distress syndrome was due to meconium stained liquor.

Present study showed that the need for NICU admission equally needed in both groups. At 38 weeks 2 babies had NICU admission. One had respiratory distress syndrome and another had congenital heart disease. At 39 weeks also 2 babies had NICU admission due to meconium aspiration syndrome. The incidence at 39 weeks babies for NICU admission was $2.1 \%$ whereas at 38 weeks it was $1.7 \%$. It was slightly increased at 39 weeks but it was not statistically significant. Chiossi et al observed that $10.3 \%$ at 38 weeks needed NICU admission and $7.7 \%$ at 39 weeks. It was comparatively high at 38 weeks than 39weeks. ${ }^{10}$ Glavind et al and Robinson et al gave almost similar reports. They concluded that there is $2 \%$ increased chance of NICU admission for baby who delivered at 38 weeks. ${ }^{13,25}$ According to Okeke et al $22.6 \%$, babies needed NICU admission $\leq 38$ weeks, whereas at 39 weeks only $1.8 \%$ babies needed NICU admission. ${ }^{23}$ But according to Wilmink et al there is only $1 \%$ increase in chance of NICU admission at 38-38+6 weeks who undergo elective LSCS. ${ }^{16}$

Although Transient Tachypnoea of Newborn is one of the most common complications for baby delivered by Elective LSCS but in our study, we did not get any case of Transient Tachypnoea of Newborn. But Zanardo et al, Robinson et al, Wilmink et al, Ghartey et al, Chiossi et al showed that the rate of chance of transient tachypnoea of newborn decreases with increase in gestational age. ${ }^{10,14,16,25}$.

According to above mentioned studies the rate of transient tachypnoea of newborn varies from $0.54 \%-4.3 \%$ at 38 weeks and $0.38 \%-2.9 \%$ at 39 weeks. Although it is very common, we did not get any case of transient tachypnoea of newborn, may be due to smaller sample size.

\section{CONCLUSION}

There was no significant maternal or neonatal complication of Elective LSCS at 38 or 39 weeks. At the same time there was no significant advantage to wait till 39 weeks to perform elective LSCS. Timing of Elective LSCS at 38 or 39 weeks had similar risk of adverse outcome. So scheduling elective LSCS at 38 weeks may be an acceptable option in women with good maternal and perinatal outcome.

The limitation of our studies are smaller study group, not following the mother and baby for long duration, improper documentation regarding cause of blood transfusion. These limitations can be overcome with large sample size, long duration of maternal and neonatal follow-up and proper documentation regarding indications of blood transfusion.

Funding: No funding sources

Conflict of interest: None declared

Ethical approval: The study was approved by the Institutional Ethics Committee

\section{REFERENCES}

1. F Gary C, Kenneth JL, Steven LB, John CH, Dwight JR, Catherine YS. Cesarean delivery and peripartum hysterectomy. In: Diane MT, George DW, Jodi SD, Barbara LH, Mala SM, James MA et al. Williams Obstetrics. $23^{\text {rd }}$ ed. New York: McGraw-Hill Medical;2010,2005,2001:544.

2. Dutta DC. Operative Obstetrics. In: Hiralal Koner, editors. Text Book of Obstetrics. $6^{\text {th }}$ ed. Culcutta:New Central Book Agency;2004:588-9 .

3. Konar H. Text book of obstetrics. $7^{\text {th }}$ ed. New central book agency publication; 2012:468-9 Kolkata.

4. Tita ATN, Landon MB, Spong CY, Lai Y, Leveno KJ, Varner MW et al. Timing of elective repeat 
cesarean delivery at term and neonatal outcomes. New Eng J Med.2009;360(2):111-20.

5. Parilla BV, Dooley SL, Jansen RD. Iatrogenic respiratory distress syndrome following elective repeat cesarean delivery. Obstet Gynecol.1993;83:392-5.

6. American College of Obstetricians and Gynecologists. Cesarean delivery on maternal request. Committee opinion no. 559. Obstet Gynecol. 2013;121:904-7.

7. Roberts CL, Nicholl MC, Algert CS, Ford JB, Morris JM, Chen JS. Rate of spontaneous onset of labour before planned repeat caesarean section at term. BMC Pregnancy and Childbirth. 2014;14:125.

8. Cohen M, Carson BS. Respiratory morbidity benefit of awaiting onset of labor after elective cesarean section. Obstet Gynecol.1985;65(6):818-24.

9. Tita ATN, Lai Y, London MB, Spong CY, Leveno KJ, Varner MW et al. Timing of elective repeat cesarean delivery at term and maternal perioperative outcomes. Obstet Gynecol. 2011;117:280-6.

10. Chiossi G, Lai Y, Landon MB, Spong CY, Rouse DJ, Varner MW. Timing of delivery and adverse outcomes in term singleton repeat cesarean deliveries. Obstet Gynecol. 2013;121(3):561-9.

11. Nahar K. Indications of caesarean section - study of 100 cases in Mymen Singh Medical College Hospital. J Shaheed Suhrawardy Medical College. 2012;1(1):6-10.

12. Roberts D, Dalziel S. Antenatal corticosteroids for accelerating fetal lung maturation for women at risk of preterm birth. Cochrane Database Syst Rev. 2006;19(3):CD004454.

13. Glavind J, Henriksen TB, Kindberg SF, Uldbjerg N. Randomised Trial of planned caesarean section prior to versus after 39 weeks: unscheduled deliveries and facility logistics - a secondary analysis. 2013;8(12):e84744.

14. Ghartey K, Coletta J, Lizarraga L, Murphy E, Ananth $\mathrm{CV}$, Gyamfi-Bannerman C. Neonatal respiratory morbidity in the early term delivery. Am J Obstet Gynecol. 2012;207(4):292.e1-4.

15. Doan E, Gibbons K, Tudehope D. The timing of elective caesarean deliveries and early neonatal outcomes in singleton infants bore 37-41 weeks gestation. ANZJOG. 2014;54:340-7 .

16. Wilmink FA, Hukkelhoven CWPM, Lunshof S, Mol BWJ, Post JAM van der, Papatsonis DNM. Neonatal outcome following elective cesarean section beyond 37 weeks of gestation: a 7-year retrospective analysis of a national registry. Am J of Obstet Gynecol. 2010;202(3):250.e1-250.e8.

17. Sperling LS, Henriksen TB, Ulrichsen H, Hedegård M, Møller $\mathrm{H}$, Hansen $\mathrm{V}$ et al. Indications for cesarean section in singleton pregnancies in two Danish counties with different cesarean section rates. Acta Obstet Gynecol Scand.1994;73(2):129-35.

18. Dahlgren LS, von Dadelszen $\mathrm{P}$, Christilaw J. Caesarean section on maternal request: risks and benefits in healthy nulliparous women and their infants. J Obstet Gynaecol Can. 2009;31:808-17.

19. Mazzoni A, Althabe F, Liu NH, Bonotti AM, Gibbons L, Sánchez AJ et al. Women's preference for caesarean section: a systematic review and metaanalysis of observational studies. BJOG. 2011;118(4):391-9.

20. Hourani M, Ziade F, Rajab M. Timing of planned caesarean section and the morbidities of the newborn. N Am J Med Sci.2011;3(10):465-8.

21. Mylonas I,FrieseK.The indications for and risks of elective cesareansection. DtschArzteblInt. 2015;112:489-95.

22. Bakr AF, Abbas MM. Severe respiratory distress in term infants born electively at high altitude. BMC Pregnancy and Childbirth. 2006;6:4.

23. Okeke TC, Onah N, Ikeako Lc, Ezenyeaku CCT, Nwogu Ikojo E. Maternal and fetal outcome of elective caesarean section at 37-38 weeks versus 39 completed weeks of gestation in Enugu, Southeast Nigeria. American Journal of Clinical Medicine Research. 2013;1(2):32-4.

24. Zanardo V, Simbi AK, Franzoi M, Soldà G, Salvadori A, Trevisanuto D. Neonatal respiratory morbidity risk and mode of delivery at term: influence of timing of elective caesarean delivery. Acta Paediatr.2004;93(5):643-7.

25. Robinson CJ, Villers MS, Johnson DD, Simpson KN. Timing of elective repeat cesarean delivery at term and neonatal outcomes: a cost analysis. Am J Obstet Gynecol. 2010;202(6);632.e1-632.e6

Cite this article as: Sebastian $\mathrm{G}$, Ghose $\mathrm{S}$, Soundararajan P. Comparison of maternal and neonatal outcome in elective lower segment cesarean section done at 38 and 39 weeks. Int J Reprod Contracept Obstet Gynecol 2017;6:1604-9. 